\title{
Energy spectra of carbon and oxygen with HELIOS E6
}

\section{Radial gradients of anomalous cosmic ray oxygen within $1 \mathrm{AU}$}

\author{
J. Marquardt ${ }^{1}$, B. Heber ${ }^{1}$, M. S. Potgieter ${ }^{2}$, and R. D. Strauss ${ }^{2}$ \\ ${ }^{1}$ Christian-Albrechts-Universität zu Kiel, Leibnizstr. 11, 24118 Kiel, Germany \\ e-mail: marquardt@physik.uni-kiel.de \\ ${ }^{2}$ Centre for Space Research, North-West-University, Potchefstroom 2520, South Africa \\ Received 3 July 2017 / Accepted 6 December 2017
}

\begin{abstract}
Context. Anomalous cosmic rays (ACRs) are well-suited to probe the transport conditions of cosmic rays in the inner heliosphere. We revisit the HELIOS data not only in view of the upcoming Solar Orbiter experiment but also to put constraints on particle transport models in order to provide new insight into the boundary conditions close to the Sun.

Aims. We present here the energy spectra of galactic cosmic ray (GCR) carbon and oxygen, as well as of ACR oxygen during solar quiet time periods between 1975 to 1977, utilizing both HELIOS spacecraft at distances between $\sim 0.3$ and 1 AU. The radial gradient $\left(G_{\mathrm{r}} \approx 50 \% / \mathrm{AU}\right)$ of $9-28.5 \mathrm{MeV}$ ACR oxygen in the inner heliosphere is about three times larger than the one determined between 1 and 10 AU by utilizing the Pioneer 10 measurements.

Methods. The chemical composition as well as the energy spectra have been derived by applying the $\mathrm{d} E / \mathrm{d} x-E$-method. In order to derive these values, special characteristics of the instrument have been taken into account.

Results. A good agreement of the GCR energy spectra of carbon and oxygen measured by the HELIOS E6 instrument between 0.3 and $1 \mathrm{AU}$ and the Interplanetary Monitoring Platform (IMP) 8 at $1 \mathrm{AU}$ was found. For ACR oxygen, we determined a radial gradient of about 50\%/AU that is three times larger than the one between 7 and $14 \mathrm{AU}$, indicating a strong change in the inner heliosphere.
\end{abstract}

Key words. Sun: heliosphere - solar-terrestrial relations - cosmic rays

\section{Introduction}

The current paradigm of anomalous cosmic rays (ACRs) can be summarized as follows: interstellar neutrals that have been swept into the heliosphere are ionized in the heliosphere to become so-called pickup ions (PUIs; Fisk et al. 1974). Because of their high ionization potentials, $\mathrm{He}, \mathrm{N}, \mathrm{O}$, and $\mathrm{Ne}$ are able to penetrate to within a few AU of the Sun before this happens (see Drews et al. 2016, and references therein). These singly ionized PUIs (Moebius et al. 1985) are subject to the Lorentz force and are therefore bound to the outward convecting heliospheric magnetic field. They become accelerated at the heliospheric termination shock (Pesses et al. 1981) and then diffuse into the heliosphere as mainly singly ionized energetic particles (Klecker et al. 1995). The crossings of the termination shock first by Voyager 1 (V1) and later by Voyager 2 (V2) have led to a new paradigm because the ACR spectra were not of the expected form (Decker et al. 2005). Several alternative acceleration models have been introduced since then (for a review, see Potgieter 2013, and references therein).

The interaction of energetic charged particles with the heliospheric magnetic field (HMF) reduces their intensities with decreasing distance to the Sun. Transport effects, in particular the adiabatic energy lose effect, yield the well known $I(E) \propto E$ proportionality for galactic cosmic rays (GCRs) at energies below about $100 \mathrm{MeV}$ nucleon $^{-1}$ (for a review, see Moraal 1993, and references therein).

Figure 1 displays the quiet-time energy spectra of hydrogen, helium, oxygen, nitrogen and carbon as measured during the 1970s solar minimum. While hydrogen shows the expected energy dependence with $I(E) \propto E^{1}$, all other elements show an upturn in the spectrum that is caused by the anomalous component that is most prominent for oxygen and nitrogen. Thus ACRs were first recognized by their different spectral shape at low energies (Hovestadt et al. 1973; Garcia-Munoz et al. 1973). The radial and latitudinal distribution of ACRs in the heliosphere can be determined by studying the evolution of the energy spectra of ions as a function of radial distance as well as latitude. The resulting radial and latitudinal gradients are therefore important to understand the transport of energetic particles in the heliosphere. Most studies concentrate on the gradients in the outer heliosphere using data from the pioneering space missions launched in the 1970s, such as Pioneer 10, Pioneer 11, V1, and V2, with the Interplanetary Monitoring Platform (IMP) 8 utilized as a baseline for their studies (see Fig. 1).

Webber et al. (1977) found a mean radial gradient of 20\%/AU with a value of 25\%/AU within $5 \mathrm{AU}$ and $10 \% / \mathrm{AU}$ from 5 to $10 \mathrm{AU}$, and of 5\%/AU beyond $10 \mathrm{AU}$ for anomalous oxygen. In a later publication, Webber et al. (1979) revised the values to $15 \% / A U$. This value was used by Trattner et al. (1995, 1996) for the 1990s $A>0$-solar magnetic epoch in order to determine the latitudinal gradients utilizing Ulysses and Solar Anomalous and Magnetospheric Particle Explorer (SAMPEX) measurements. However, Cummings et al. (1990, 1995) found the radial gradient to depend on the radial distance as $r^{-0.96}$ and $r^{-1.7}$ for an $A<0$ and $A>0$ solar magnetic epoch, respectively. Recent determinations of the radial gradient were performed by Cummings et al. (2009) for an $A<0$-magnetic epoch utilizing 


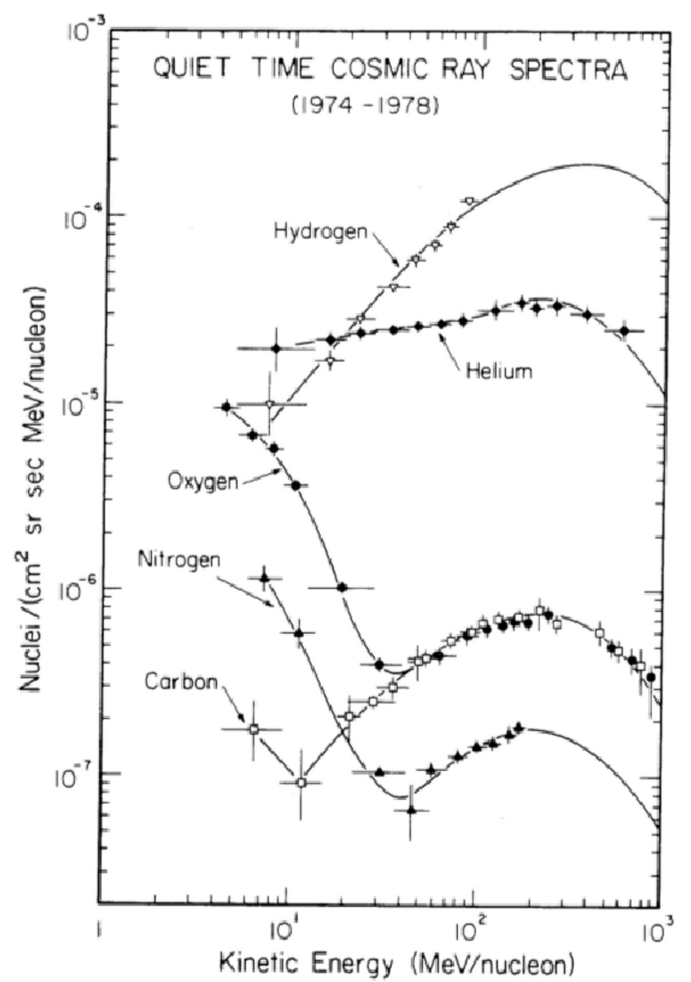

Fig. 1. Quiet-time energy spectra for the elements $\mathrm{H}, \mathrm{He}, \mathrm{C}, \mathrm{N}$, and O measured at $1 \mathrm{AU}$ over the period from 1974 to 1978 . We note the "anomalous" enhancements in the low-energy spectra of $\mathrm{He}, \mathrm{N}$, and $\mathrm{O}$ (Fig. 1.2 of Christian 1989).

the measurements from the low-energy telescopes aboard the Solar Terrestrial Relations Observatory (STEREO) A and B and Ulysses leading to a radial gradient of $45 \% / \mathrm{AU}$ and $51 \% / \mathrm{AU}$ at 4.5 to 15 and 7.3 to $15.6 \mathrm{MeV}$ nucleon $^{-1}$, respectively. Because of the strong dependence of the radial gradient on distance, larger radial gradients are expected in the inner than in the outer heliosphere. The only future measurements of ACR oxygen within the inner heliosphere will be performed by the Parker Solar Probe (Fox et al. 2016). However, recently we showed that the HELIOS E6 detector (Kunow 1981) is capable of separating the chemical composition up to neon (Marquardt et al. 2015). Therefore, we revisit here the energetic particle measurements gathered by the HELIOS experiment during the solar minimum from 1974 to 1977. In what follows, we apply the pulse height analysis developed by Marquardt et al. (2015) in order to determine reliable energy spectra for carbon and oxygen to infer the non-local differential radial gradient for ACR oxygen in the inner heliosphere between $\sim 0.3$ and $1 \mathrm{AU}$.

\section{HELIOS and experiment 6 (E6)}

HELIOS A and HELIOS B were launched on December 10, 1974 and January 15, 1976, respectively. The two almost identical space probes were sent into ecliptic orbits around the Sun. The orbital period around the Sun was 190 days for HELIOS A and 185 days for HELIOS B, and their perihelia were 0.3095 and 0.290 AU, respectively. The upper panels of Figs. 2 and 3 display the radial distance of HELIOS A and B from the launch of HELIOS A in 1974 to the end of 1978, receptively. Marked by the green and blue shaded regions are the close and far periods for which the spacecraft were within $0.45 \mathrm{AU}$ and beyond 0.9 AU from the Sun. The lower panels of both figures indicate

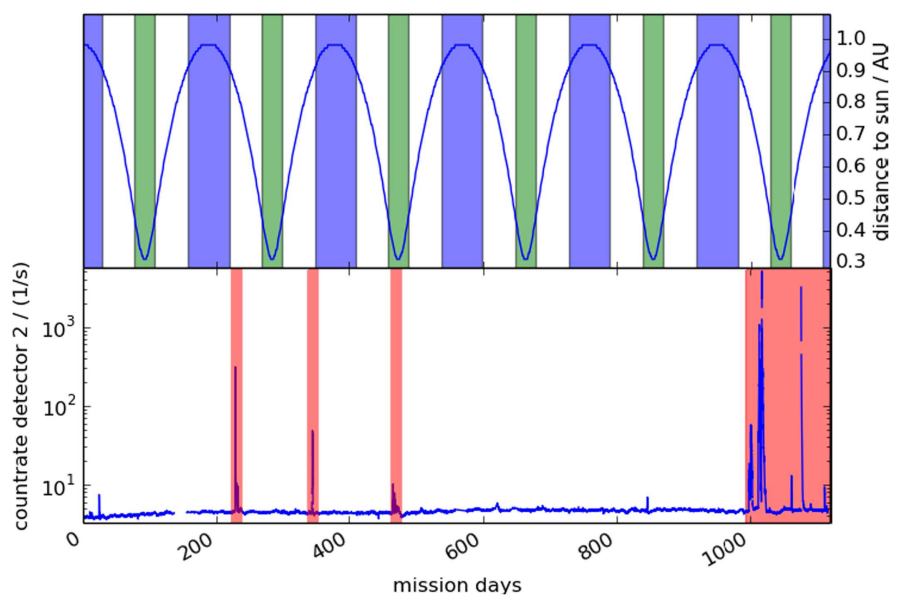

Fig. 2. Upper panel: radial distance of the HELIOS A spaceprobe from the Sun. Indicated by the green and blue shaded regions are the close and far periods for which the spacecraft was within 0.3 and $0.45 \mathrm{AU}$, and 0.9 and $1 \mathrm{AU}$, respectively. Lower panel: count rate of $Z \geq 2$ ions with energies from about 4 to $13 \mathrm{MeV}$ nucleon $^{-1}$ for helium. Shaded periods have been omitted in our analyses.

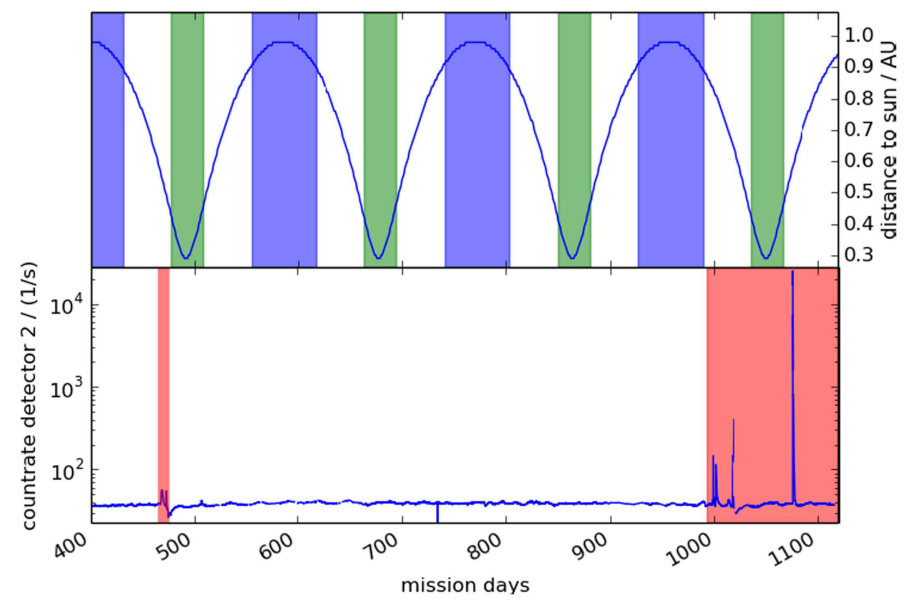

Fig. 3. Similar to Fig. 2, but for HELIOS 2.

the hourly averaged count rate of 4 to $13 \mathrm{MeV}$ nucleon $^{-1}$ ions with $z \geq 2$. Time periods that indicate intensity increases due to solar energetic particle events are indicated by the red shaded regions and have been omitted in our analysis.

The Kiel experiment, E6, has been described in detail by Kunow (1981). It is one of three particle detectors aboard HELIOS that allowed the study of energetic particles in the energy range from $1.3 \mathrm{MeV}$ nucleon $^{-1}$ to above $1000 \mathrm{MeV}$ nucleon $^{-1}$ for ions and from 0.3 to $8 \mathrm{MeV}$ for electrons. Recently, Marquardt et al. (2015) showed that the instrument is capable of determining the chemical composition from hydrogen to neon in the energy range from a few to several tenths of a MeV nucleon ${ }^{-1}$.

The detector system is sketched in Fig. 4 and consists of five semi-conductor detectors of increasing thickness indicated in the figure; while the first two detectors, D1(A) and D2(B), are silicon-surface-barrier detectors with a thickness of 100 and $1000 \mu \mathrm{m}$, the other three are lithium drifted detectors with a thickness of $3000 \mu \mathrm{m}$. The first two (D1 and D2) are used for determining the lowest energy channels for the definition of the geometry factor for stopping particles (energy ranges 


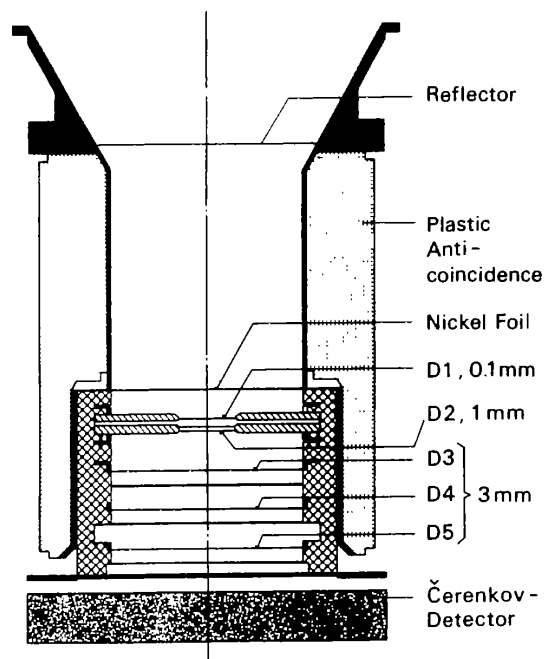

Fig. 4. Schematic of the HELIOS E6 (adapted from Marquardt et al. 2015).

below $51 \mathrm{MeV}$ nucleon $^{-1}$ ), as well as for discriminating between electrons and ions. Since the thickness of $100 \mu \mathrm{m}$ does not allow us to measure minimum ionizing particles, electrons are separated from ions by the following trigger conditions:

- no signal in the first detector $\longrightarrow$ electron;

- signal in the first detector $\longrightarrow$ ion.

To avoid false identifications by these discrimination conditions, the first and second detectors are as close as possible to each other. Charged particles, which penetrate the fifth detector and the aluminum absorber beneath, are detected in the SapphireCerenkov-detector. The Cerenkov-threshold for this material $(n=1.8)$ is $E_{\mathrm{s}}=210 \mathrm{MeV}$ nucleon $^{-1}$. Because the SapphireCerenkov-detector also delivers scintillation light, particles in the energy range above $51 \mathrm{MeV}$ nucleon $^{-1}$ are included in an integral channel.

\section{Data correction and analysis}

The E6 particle telescope relies on the $\mathrm{d} E / \mathrm{d} x-E$-method (see e.g. McDonald \& Ludwig 1964). In order to interpret the measured data, a detailed understanding of the instrument is needed and can be obtained by modelling the physical processes inside the detector taking into account the instrument geometry as well as the environment (e.g. Heber et al. 2005; Marquardt et al. 2015). We need to correct for the so-called edge effects. Due to the decreasing charge collecting efficiency, from a certain radius outward the measured energy loss is spatially-dependent. This effect is particularly severe for the first two detectors, as described by Marquardt et al. (2015). Figure 5 displays the energy spectra of carbon (magenta symbols) and oxygen (black and red symbols) measured by HELIOS E6, binned in the same way as the energy spectra shown in Fig. 1. For oxygen we were able to restrict the time period under investigation to the periods when the spacecraft was beyond 0.9 AU. Due to the limited counting statistics, the whole time period, including periods when the spacecraft was close to the Sun, has to be used for carbon. The values for carbon (blue symbols) and oxygen (green symbols) from Fig. 1 have been added, indicating a reasonable agreement between the HELIOS and IMP observations. We note that for oxygen we utilized time periods when the spacecraft was beyond 0.9 AU. Due to the reduced statistics we need to take into account all periods for carbon, including the ones

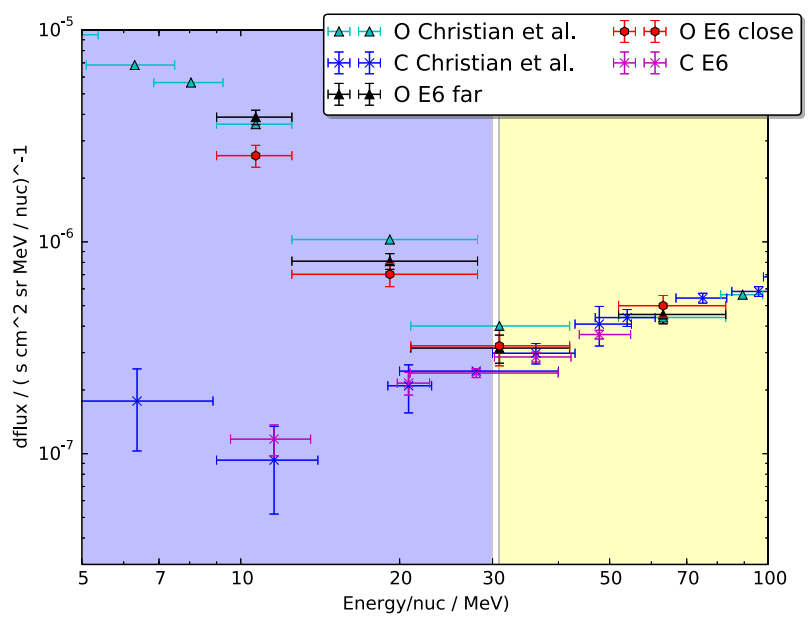

Fig. 5. Energy spectra of carbon and oxygen measured by the E6 in comparison with the data taken from Fig. 1.

when HELIOS is within 0.9 AU. Therefore, we utilize only the HELIOS measurements in what follows. In order to determine radial gradients in the inner heliosphere, we divide the radial distances from the Sun into a set of measurements obtained during all far (1.0-0.9 AU) and close (0.45-0.3 AU) time periods (see Figs. 2 and 3). Since an upturn in the energy spectra is notable for energies below $30 \mathrm{MeV}$ nucleon $^{-1}$, oxygen ions below and above $30 \mathrm{MeV}^{\text {nucleon }}{ }^{-1}$ are classified as ACRs and GCRs, respectively. For carbon, the total number of observed particles is insufficient to determine a radial gradient.

\section{Results}

Table 1 and Fig. 5 summarize the oxygen fluxes measured by HELIOS E6. The fluxes for oxygen are given in the same energy ranges as for IMP 8 (see Fig. 1). We use the fluxes during the far periods as a proxy for the $1 \mathrm{AU}$ measurements.

Table 1 provides the oxygen fluxes in the energy range 9-28 MeV nucleon ${ }^{-1}$, similar to the energy range of 19-23.5 MeV nucleon ${ }^{-1}$ used by Webber et al. (1981). Assuming that temporal changes of the ACR oxygen flux are smaller than radial changes, we can calculate the corresponding radial gradient, $G_{\mathrm{r}}$, as

$G_{\mathrm{r}}=\ln \left(\frac{I_{\text {far }}}{I_{\text {close }}}\right) \frac{1}{\Delta r}$

using $\Delta r=0.6$ AU. These gradients are summarized in Table 2. The uncertainties given in the table take into account the statistical uncertainties only.

For GCR oxygen the ratio $I_{\text {far }} / I_{\text {close }}$ is consistent with unity, thus implying a very small radial gradient. Webber et al. (1981) investigated the radial gradients of ACR oxygen measured from 1972 to 1980 by the Pioneer 10 and IMP spacecraft. They showed that the radial gradient had only slight variations from 1974 to 1977 when Pioneer 10 moved from about 7 AU to about 14 AU. In their Fig. 2 they give a mean value of $G_{\mathrm{r}}=15 \% / \mathrm{AU}$. The upper panel of Fig. 6 shows the Pioneer 10 to IMP ratio in the energy range between 9 and $28.5 \mathrm{MeV}$ nucleon $^{-1}$ as a function of radial distance for the solar minimum period of solar cycle 22 . The data are taken from (Webber et al. 1981) Fig. 2, and indicated on the figure by magenta triangles. In addition, the HELIOS close to far ratio, at $\sim 0.38 \mathrm{AU}$, obtained during the time span from 1975 to 
Table 1. Oxygen (O) fluxes $I_{\text {far }}$ and $I_{\text {close }}$ and their relative uncertainty averaged over all time periods marked in Figs. 2 and 3 for time periods when the spacecraft distance were $>0.9$ and $<0.45$ AU, respectively.

\begin{tabular}{lllllll}
\hline \hline Element & $\begin{array}{l}E_{1} \\
\mathrm{MeV} \mathrm{nuc}^{-1}\end{array}$ & $\begin{array}{l}E_{2} \\
\mathrm{MeV} \mathrm{nuc}^{-1}\end{array}$ & $\begin{array}{l}I \text { (far) } \\
\mathrm{p} \mathrm{cm}^{-2} \mathrm{sr} \mathrm{s} \mathrm{MeV} \mathrm{nuc}^{-1}\end{array}$ & $\begin{array}{l}\Delta I \\
\%\end{array}$ & $\begin{array}{l}I \text { (close) } \\
\mathrm{p} \mathrm{cm}^{-2} \mathrm{sr} \mathrm{s} \mathrm{MeV} \mathrm{nuc}^{-1}\end{array}$ & $\begin{array}{c}\Delta I \\
\%\end{array}$ \\
\hline $\mathrm{O}$ & 9 & 13 & $3.88 \times 10^{-6}$ & 7 & $2.55 \times 10^{-6}$ & 11 \\
$\mathrm{O}$ & 13 & 28 & $0.811 \times 10^{-6}$ & 8 & $0.703 \times 10^{-6}$ & 12 \\
$\mathrm{O}$ & 21 & 48 & $0.315 \times 10^{-6}$ & 15 & $0.323 \times 10^{-6}$ & 19 \\
$\mathrm{O}$ & 52 & 83 & $0.454 \times 10^{-6}$ & 9 & $0.500 \times 10^{-6}$ & 11 \\
\hline $\mathrm{O}$ & 9 & 28.5 & $1.37 \times 10^{-6}$ & 6 & $1.04 \times 10^{-6}$ & 4 \\
$\mathrm{O}$ & 30 & 85 & $0.435 \times 10^{-6}$ & 4 & $0.459 \times 10^{-6}$ & 3 \\
\hline
\end{tabular}

Notes. The first two columns give the lower and upper energy limits.

Table 2. Average radial gradients $G_{\mathrm{r}}$ and their lower and upper limits, in the last two columns, for the energy range summarized in Table 1.

\begin{tabular}{llllll}
\hline \hline Element & $E_{1}$ & $E_{2}$ & $G_{\mathrm{r}}$ & $G_{\mathrm{r}}^{\mathrm{l}}$ & $G_{\mathrm{r}}^{\mathrm{u}}$ \\
& $\mathrm{MeV} \mathrm{nuc}^{-1}$ & $\mathrm{MeV} \mathrm{nuc}^{-1}$ & $\% / \mathrm{AU}$ & $\% / \mathrm{AU}$ & $\% / \mathrm{AU}$ \\
\hline $\mathrm{O}$ & 9 & 13 & 73 & 50 & 99 \\
$\mathrm{O}$ & 13 & 28 & 25 & 0 & 54 \\
$\mathrm{O}$ & 21 & 48 & -4 & -43 & 45 \\
$\mathrm{O}$ & 52 & 83 & -17 & -41 & 12 \\
\hline $\mathrm{O}$ & 9 & 28.5 & 48 & 36 & 60 \\
$\mathrm{O}$ & 30 & 85 & -10 & -20 & 0 \\
\hline
\end{tabular}

mid 1977, is included as the black square. The middle and lower panels of the figure show the radial gradient, calculated by using Eq. (1), as a function of time and distance. From this figure it is evident that a strong increase with decreasing distance in the radial gradient must occur in the inner heliosphere within $2 \mathrm{AU}$.

The red triangles in the lower panel of Fig. 6 show the results of the computations described by Strauss \& Potgieter (2010). Whereas the computations show an increase of the ACR-oxygen gradient from $14 \mathrm{AU}$ inwards, peaking between 2 and $3 \mathrm{AU}$, the Pioneer 10 measurements show a nearly constant radial gradient of about 10 to $15 \% / \mathrm{AU}$ from 3 to $14 \mathrm{AU}$, but the gradient then follows the predicted trend beyond 10 AU. However, the HELIOS measurements indicate a larger radial gradient that is in good agreement with the maximum gradient of the model, although much closer to the Sun. Pioneer 10 measurements between 7 to 14 AU coincide with the time of the HELIOS observations.

The results shown in Fig. 6 therefore indicate that ACR oxygen can easily penetrate into the inner heliosphere within $0.6 \mathrm{AU}$. The fact that the model and observations agree beyond $\sim 10 \mathrm{AU}$ is indeed encouraging, even more so that the maximum value of the gradient, $G_{\mathrm{r}}^{\max } \sim 45 \% / \mathrm{AU}$, also seems to be consistent with the model prediction.

The quantitative differences between the model and observations should, however, be investigated in detail in future. Reasons for the disagreement may include: (1) Since the Pioneer observations within $7 \mathrm{AU}$ were obtained during a different phase of the solar cycle, these measurements may be influenced by temporal effects, while the computations are solutions of a steady-state model. (2) Near the Sun, and especially within 1 AU, the inner boundary condition assumed in the model may influence the calculated gradient (see also the discussion by Strauss \& Potgieter 2010). (3) The assumed Parker (1965) transport equation might
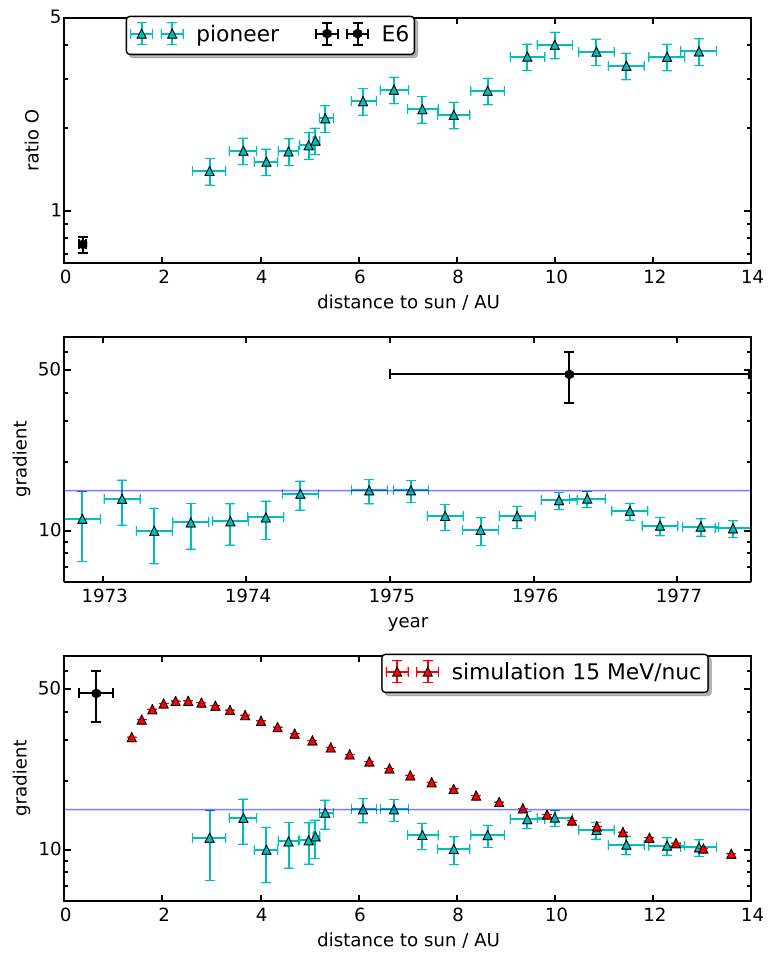

Fig. 6. Upper panel: radial dependence of the ACR oxygen flux ratio in the energy range between 9 and $23.5 \mathrm{MeV}$ nucleon ${ }^{-1}$. Data beyond $1 \mathrm{AU}$ are taken from Webber et al. (1981). Middle and lower panels: temporal and radial variation of the radial gradient. The red symbols show the computations from the modelling of Strauss \& Potgieter (2010).

not be valid closer to the Sun as magnetic focussing can lead to an anisotropic ACR distribution. (4) Ultimately, the computed gradients depend on the diffusion and drift coefficients as assumed in the model. They, on their part, are based on the underlaying assumed turbulence, diffusion, and drift theories, which may need to be refined for ACR oxygen by observance of the new measurements presented here.

\section{Summary and conclusion}

Marquardt et al. (2015) showed that the HELIOS E6 experiment is well suited for measuring the chemical composition of particles from carbon to neon. Here, we determined the quiettime spectrum of carbon and oxygen in the energy range from 
$9 \mathrm{MeV}$ nucleon $^{-1}$ to above $80 \mathrm{MeV}$ nucleon $^{-1}$. A good agreement between the HELIOS and the IMP measurements, as reported by Christian (1989), was found. In order to compute the radial gradient in the inner heliosphere, the data set was divided into a period when the spacecraft was within $0.45 \mathrm{AU}\left(I_{\text {close }}\right)$ and another period with distances greater than $0.9 \mathrm{AU}\left(I_{\mathrm{far}}\right)$.

For GCR oxygen with energies between 30 and $80 \mathrm{MeV}$ nucleon $^{-1}$, the ratio between the fluxes measured close to and far from the Sun is consistent with $1.0 \pm 0.1$, indicating no significant radial gradient. However, for ACR oxygen, a significant radial gradient of $G_{\mathrm{r}}=73_{-23}^{+26} \% / \mathrm{AU}$ and $G_{\mathrm{r}}=25_{-25}^{+29} \% / \mathrm{AU}$ was found for 9 to 13 and 13 to $28 \mathrm{MeV}$ nucleon $^{-1}$. By assuming a spectral shape of $I(E) \propto E^{1}$ for the GCRs and subtracting this GCR contribution from the flux in the 13 to $28 \mathrm{MeV}_{\text {nucleon }}{ }^{-1}$ range, the gradient increases to $G_{\mathrm{r}}=40 \% / \mathrm{AU}$. However, due to the uncertainties of this method a detailed error estimation is beyond the scope of this work.

When using the whole energy range from 9 to 28.5 MeV nucleon ${ }^{-1}$, we find $G_{\mathrm{r}}=48_{-12}^{+12} \% / \mathrm{AU}$. This energy range was used also by Webber et al. (1981) to determine the radial dependence of $G_{\mathrm{r}}$ beyond 1 AU. The radial gradient that we report here is about three times larger than the one determined between 1 and $10 \mathrm{AU}$ by utilizing the Pioneer 10 measurements.

Comparing results from these Pioneer 10 measurements to HELIOS results, we find that the radial gradient of ACR oxygen significantly increases within the first 2 AU from the Sun. Evidently, ACR oxygen, with a rigidity much larger than GCR oxygen, may penetrate the heliosphere to come very close to the Sun, exhibiting in the process a very steep gradient. Future information to validate our results will come from the Parker Solar Probe.

Acknowledgements. The work was partly carried out within the framework of the bilateral BMBF-NRF-project "Astrohel" (01DG15009) as supported by the Bundesministerium für Bildung und Forschung (BMBF) and the South African National Research Foundation (NRF).

\section{References}

Christian, E. R. 1989, PhD thesis, California Institute of Technology, Pasadena Cummings, C. A., Mewaldt, A. R., Stone, C. E., \& Webber, R. W. 1990, Int. Cosmic Ray Conf., 6, 206

Cummings, A. C., Mewaldt, R. A., Blake, J. B., et al. 1995, Geophys. Res. Lett., 22,341

Cummings, A. C., Tranquille, C., Marsden, R. G., Mewaldt, R. A., \& Stone, E. C. 2009, Geophys. Res. Lett., 36, L18103

Decker, R. B., Krimigis, S. M., Roelof, E. C., et al. 2005, Science, 309, 2020

Drews, C., Berger, L., Taut, A., \& Wimmer-Schweingruber, R. F. 2016, A\&A, 588, A12

Fisk, L. A., Kozlovsky, B., \& Ramaty, R. 1974, ApJ, 190, L35

Fox, N. J., Velli, M. C., Bale, S. D., et al. 2016, Space Sci. Rev., 204, 7

Garcia-Munoz, M., Mason, G. M., \& Simpson, J. A. 1973, ApJ, 182, L81

Heber, B., Kopp, A., Fichtner, H., \& Ferreira, S. E. S. 2005, Adv. Space Res., 35 , 605

Hovestadt, D., Vollmer, O., Gloeckler, G., \& Fan, C. Y. 1973, Int. Cosmic Ray Conf., 2, 1498

Klecker, B., McNab, M. C., Blake, J. B., et al. 1995, ApJ, 442, L69

Kunow, H. 1981, Cosmis ray experiment on board the solar probes Helios-1 and 2 (experiment no. 6), Tech. Rep.

Marquardt, J., Heber, B., Hörlöck, M., Kühl, P., \& Wimmer-Schweingruber, R. F. 2015, J. Phys. Conf. Ser., 632, 012016

McDonald, F. B., \& Ludwig, G. H. 1964, Phys. Rev. Lett., 13, 783

Moebius, E., Hovestadt, D., Klecker, B., Scholer, M., \& Gloeckler, G. 1985, Nature, 318, 426

Moraal, H. 1993, Nucl. Phys. B Proc. Suppl., 33, 161

Parker, E. N. 1965, Planet. Space Sci., 13, 9

Pesses, M. E., Eichler, D., \& Jokipii, J. R. 1981, ApJ, 246, L85

Potgieter, M. S. 2013, Liv. Rev. Sol. Phys., 10, 3

Strauss, R. D., \& Potgieter, M. S. 2010, J. Geophys. Res. (Space Phys.), 115, A 12111

Trattner, K. J., Marsden, R. G., Sanderson, T. R., et al. 1995, Geophys. Res. Lett., 22,337

Trattner, K. J., Marsden, R. G., Bothmer, V., et al. 1996, A\&A, 316, 519

Webber, W. R., McDonald, F. B., \& Trainor, J. H. 1977, Int. Cosmic Ray Conf., 3, 233

Webber, W. R., McDonald, F. B., Trainor, J. H., \& von Rosenvinge, T. T. 1979, Int. Cosmic Ray Conf., 5, 353

Webber, W. R., McDonald, F. B., von Rosenvinge, T. T., \& Mewaldt, R. A. 1981, Int. Cosmic Ray Conf., 10, 92 